\title{
Carbon burning rates on the compound nucleus formation
}

\author{
Masahiko Katsuma ${ }^{1,2, *}$ \\ ${ }^{1}$ Advanced Mathematical Institute, Osaka City University, Osaka 558-8585, Japan \\ ${ }^{2}$ Institut d'Astronomie et d'Astrophysique, Université Libre de Bruxelles, B-1050 Brussels, Belgium
}

Abstract. The ${ }^{12} \mathrm{C}+{ }^{12} \mathrm{C}$ reaction rates based on the compound nucleus formation seem to be concordant with the standard rates. The resonant contribution in ${ }^{12} \mathrm{C}+{ }^{12} \mathrm{C}$ is also discussed. To put the rates on firm ground, the resonances below $E_{c . m .}=3 \mathrm{MeV}$ will have to be studied further.

\section{Introduction}

The ${ }^{12} \mathrm{C}+{ }^{12} \mathrm{C}$ fusion reaction is one of the key reactions to understand the evolution of massive stars and various explosive scenarios. However, precise measurements of cross sections below $E_{c . m .}=3 \mathrm{MeV}$ are difficult because of the tiny amplitudes caused by the Coulomb barrier. At present, the direct measurements have been performed in $E_{c . m .}=2.1-2.5 \mathrm{MeV}$ [1]. The indirect measurements have been studied with ${ }^{24} \operatorname{Mg}\left(\alpha, \alpha^{\prime}\right)$ [2] and Trojan horse method [3]. The derived rates [3] are much faster than CF88 [4], due to the resonant states at $E_{\text {c.m. }} \approx 1.5 \mathrm{MeV}$, which may have the ${ }^{12} \mathrm{C}+{ }^{12} \mathrm{C}$ molecule-like structure. The nuclear fusion for ${ }^{12,13} \mathrm{C}+{ }^{13} \mathrm{C}$ have also been discussed experimentally to understand $\mathrm{C}+\mathrm{C}$ comprehensively [5].

In this presentation, I use a barrier penetration model (BPM), and I show the calculated results of isotope dependence of fusion cross sections and reaction rates in $\mathrm{C}+\mathrm{C}$. The transmission coefficients are given by the WKB approximation, semi-classically, and the potentials used in the present work are calculated from a single-folding model [6] with [7, 8]. I also discuss the contribution from the resonances in ${ }^{12} \mathrm{C}+{ }^{12} \mathrm{C}$ by comparing the result of BPM with a schematic calculation of the coupled-channels multi-level $R$-matrix [9].

\section{Compound nucleus formation}

Before moving on to the results, let me recall the compound nucleus $(\mathrm{CN})$ formation, to understand the reaction mechanism in $\mathrm{C}+\mathrm{C}$. The ${ }^{12} \mathrm{C}+{ }^{12} \mathrm{C}$ potential obtained from the studies of elastic scattering has predicted the sequences of the rotational excitation in ${ }^{24} \mathrm{Mg}[7,10]$. These resonances are the excited states with the ${ }^{12} \mathrm{C}+{ }^{12} \mathrm{C}$ molecule-like structure in ${ }^{24} \mathrm{Mg}$. However, the potential resonances at $E_{c . m .} \approx 0$ are dispersed easily, because of the couplings to reaction channels. Although the inelastic channels are closed at $E_{c . m} .=4.44 \mathrm{MeV}$, other reaction channels are open, and they work as absorption to the entrance channel. Accordingly, their fragments are distributed around the original energy positions. In fact, many fragments of $J^{\pi}=2^{+}, 4^{+}$resonances have been observed, in addition to $0^{+}$[3]. Whereas most of flux are consumed by Coulomb scattering, a small amount of flux is captured into the longliving fragment levels, and exits through the proton, neutron, and $\alpha$ channels after forming a compound nucleus. Under the circumstance, the reactions should be described statistically,

\footnotetext{
*e-mail: mkatsuma@gmail.com
} 

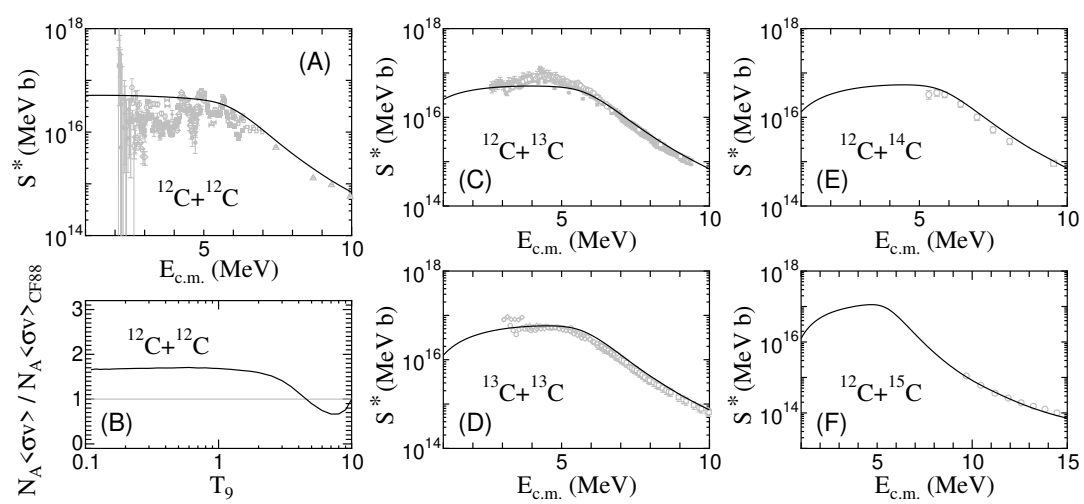

Figure 1. Comparison in $S^{*}$ between BPM and experimental data: (A) ${ }^{12} \mathrm{C}+{ }^{12} \mathrm{C}$, (C) ${ }^{12} \mathrm{C}+{ }^{13} \mathrm{C}$, (D) ${ }^{13} \mathrm{C}+{ }^{13} \mathrm{C}$, (E) ${ }^{12} \mathrm{C}+{ }^{14} \mathrm{C}$, and $(\mathrm{F}){ }^{12} \mathrm{C}+{ }^{15} \mathrm{C}$. The solid curves are the results with BPM. The experimental data are taken from $[1,5,11,12]$. (B) The derived rates of ${ }^{12} \mathrm{C}+{ }^{12} \mathrm{C}$ are shown in ratio of CF88 [4].

and the emitted nuclei have to be treated as evaporation products. Therefore, I adopt BPM and $R$-matrix based on the $\mathrm{CN}$ formation in the present study.

In BPM, the energy-averaged fusion cross sections are given by $\sigma_{F}=\left(\pi / k^{2}\right) \sum_{L}(2 L+$ $1)\left\langle\left|S_{C N}\right|^{2}\right\rangle . k$ is the wavenumber; $L$ is the angular momentum between nuclei. $\left\langle\left|S_{C N}\right|^{2}\right\rangle$ are the transmission coefficients $T_{L}$, given by WKB approximation for $E_{c . m}$. $<E_{B}: T_{L}=\{1+$ $\left.\exp \left[2 \int_{R_{1}}^{R_{2}} K(R) d R\right]\right\}^{-1}, K(R)=\left\{(2 \mu) / \hbar^{2}\left[\tilde{U}_{L}(R)-E_{c . m .}\right]\right\}^{1 / 2} . E_{B}$ is the barrier height energy. $\mu$ is the reduced mass. $R_{1}$ and $R_{2}$ are the inner and outer turning points of effective potentials $\tilde{U}_{L}$. The nuclear potentials in $\tilde{U}_{L}$ are calculated recursively from the single-folding model [6-8]. The cross sections are also given by $R$-matrix theory, $\sigma_{F}=\left(\pi / k^{2}\right) \sum_{c L}(2 L+1)\left|S_{c, 0}^{L}\right|^{2}$. $S_{c, 0}^{L}$ is the $S$-matrix deduced from the $R$-matrix with the resonance parameters in [3]. The reduced width of ${ }^{12} \mathrm{C}+{ }^{12} \mathrm{C}$ is statistically assumed to be a constant $\gamma_{i L}^{2}=0.001 \gamma_{W}^{2}$ for all levels, based on the $\mathrm{CN}$ formation. $\gamma_{W}^{2}$ is the Wigner limit. In [5], the transmission coefficients are calculated from an approximation using the unitarity relation of $S$-matrix. To display the cross sections, the $S^{*}$ factors are defined as $S^{*} \equiv \sigma_{F} E_{c . m .} \exp \left(87.21 E_{c . m}^{-1 / 2}+0.46 E_{c . m .}\right)$, e.g. [3].

\section{Results \& Conclusion}

The calculated $S^{*}$ factors with BPM for ${ }^{12} \mathrm{C}+{ }^{12} \mathrm{C}$ are shown by the solid curve in Fig. 1(A), and they appear to give the trend of the energy variation in the experimental data $[1,11]$. The derived reaction rates are shown in ratio to CF88. (Fig. 1(B)) They seem consistent with CF88. For ${ }^{12,13} \mathrm{C}+{ }^{13} \mathrm{C}$ and ${ }^{12} \mathrm{C}+{ }^{14,15} \mathrm{C}$, the present calculations of BPM (solid curves) reproduce the experimental data $[5,12]$, consistently, as shown in Figs. 1(C) - 1(F).

Figure 2(A) illustrates the isotope dependence of $S^{*}$ obtained from BPM. In BPM, the ${ }^{12} \mathrm{C}+{ }^{12} \mathrm{C} S^{*}$ factors below $E_{c . m .}=2 \mathrm{MeV}$ become the largest, so the derived reaction rates are the fastest below $T_{9}=0.8$ (Fig. 2(B)). In addition, the $S^{*}$ factors are found to be enhanced at the sub-barrier energies as the number of neutrons increases. Particularly, those of ${ }^{12} \mathrm{C}+{ }^{15} \mathrm{C}$ are enhanced larger. The barrier radius $R_{B}$ and barrier height energy $E_{B}$ in the present calculations are shown in Figs. 2(C) and 2(D), as a function of the mass number. $R_{B}\left(E_{B}\right)$ becomes large (small) as the number of neutrons increases. Especially, $R_{B}$ suddenly becomes large at ${ }^{15} \mathrm{C}$. This is caused by the weakly-bound $s$-wave neutron in ${ }^{15} \mathrm{C}$. Therefore, the corresponding $S^{*}$ and reaction rates are expected to be enhanced more by the sharp reduction of $E_{B}$.

The resonant contribution in ${ }^{12} \mathrm{C}+{ }^{12} \mathrm{C}$ is shown in Fig. 3. From the result of the $R$-matrix calculation by the solid curve in Fig. 3(A), the values of $S^{*}$ are found to be much smaller than those of [3]. In the present calculation, I include the same 34 levels and four exit channels as 

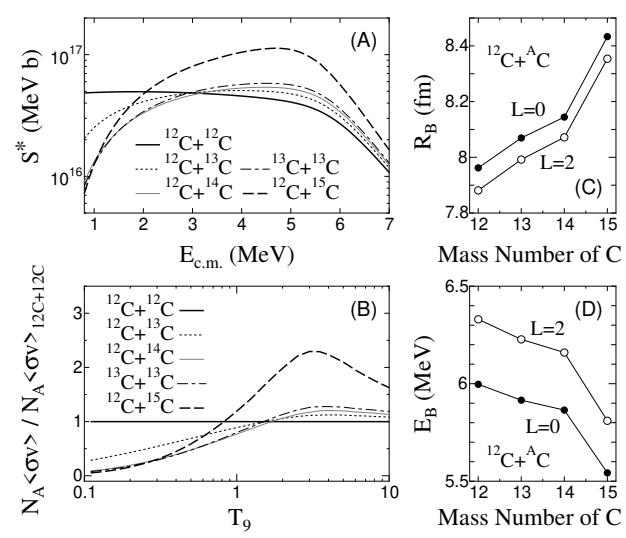

Mass Number of C

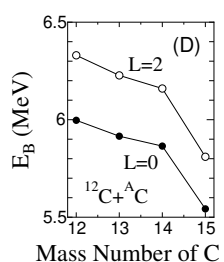

Figure 2. Isotope dependence of (A) $S^{*}$, (B) reaction rates, (C) $R_{B}$, and (D) $E_{B}$, obtained from BPM. $(\mathrm{A}, \mathrm{B})$ The curves are the results for the respective system shown in figure. (C,D) $R_{B}$ and $E_{B}$ are obtained from $\tilde{U}_{L}$. The solid and open circles are the values for $L=0$ and $L=2$, respectively.
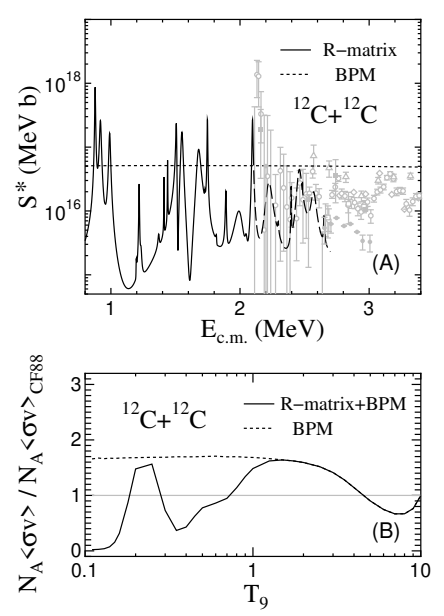

Figure 3. (A) Low-energy extrapolation of $S^{*}$ and (B) the reaction rates. The solid and dotted curves are the results calculated from $R$-matrix and BPM. The experimental data are taken from $[1,11]$.

those in [3]. If $\gamma_{i L}^{2}=0.05 \gamma_{W}^{2}$ is used as the reduced width of ${ }^{12} \mathrm{C}+{ }^{12} \mathrm{C}$ at $E_{c . m} \approx 1.5 \mathrm{MeV}$, the reaction rates would increase like those in [3]. The carbon burning rates are sensitive to the reduced width of ${ }^{12} \mathrm{C}+{ }^{12} \mathrm{C}$. In addition, the reaction rates estimated from the $R$-matrix extrapolation are confirmed to be reduced from the result of BPM. However, the derived rates at $T_{9}=0.6$ still seem to be consistent with $\mathrm{CF} 88$. To put the rates on firm ground, the resonances below $E_{c . m .}=3 \mathrm{MeV}$ will have to be scrutinized further.

\section{Acknowledgements}

I thank Prof. X.D. Tang for his valuable comments and providing me with the experimental data.

\section{References}

[1] W.P. Tan et al., PRL 124, 192702 (2020); G. Fruet et al., PRL 124, 192701 (2020); C.L. Jiang et al., PRC 97, 012801(R) (2018); T. Spillane et al., PRL 98, 122501 (2007)

[2] T. Kawabata et al., J. Phys. Conf. 436, 012009 (2013)

[3] A. Tumino et al., Nature 557, 687 (2018); Springer Proc. Phys. 219, 17 (2019)

[4] G.R. Caughlan, W.A. Fowler, Atom. Data Nucl. Data Tables 40, 283 (1988)

[5] M. Notani et al., PRC 85, 014607 (2012); X.D. Tang, Springer Proc. Phys. 219, 13 (2019); B.B. Back et al., Rev. Mod. Phys. 86, 317 (2014)

[6] H. Nishioka et al., Nucl. Phys. A 415, 230 (1984)

[7] Y. Kondō, M. Katsuma, Nucl. Phys. A 738, 226 (2004); Y. Kondō, M.E. Brandan, G.R. Satchler, Nucl. Phys. A 637, 175 (1998)

[8] B.A. Watson et al., Phys. Rev. 182, 977 (1969)

[9] A.M. Lane, R.G. Thomas, Rev. Mod. Phys. 30, 257 (1958)

[10] M.E. Brandan, G.R. Satchler, Phys. Rep. 285, 143 (1997)

[11] E.F. Aguilera et al., PRC 73, 064601 (2006); D.G. Kovar et al., PRC 20, 1305 (1979); B. Dasmahapatra et al., Nucl. Phys. A 384, 257 (1982); M.D. High, B. Čujec, Nucl. Phys. A 282, 181 (1977); H.W. Becker et al., Z. Phys. A 303, 305 (1981)

[12] EXFOR, International Network of Nuclear Reaction Data Centres (NNDC); Nuclear Reaction Video (NRV) project supported by Russian Foundation for Basic Research 